\title{
Reconstruction From Multiple Poses in Fluorescence Imaging: Proof of Concept
}

\author{
Denis Fortun, Member, IEEE, Paul Guichard, Ning Chu, Member, IEEE, and Michael Unser, Fellow, IEEE
}

\begin{abstract}
One disadvantage of all fluorescence imaging modalities is a poor axial resolution. To overcome this issue, we propose a novel approach to reconstruct fluorescence volumes with isotropic high resolution, from images of particle replicates observed at multiple orientations. We design a joint deconvolution-and-multiview reconstruction approach dedicated to three-dimensional fluorescence imaging. We address the computational challenge associated to big data by designing a fast augmented-Lagrangian optimizer. The computational cost of the iterative part of the algorithm does not depend on the number of input particles. We experimentally demonstrate the resolution improvement yielded by our framework and its ability to handle practical constraints like large PSF sizes and large number of particles. The validation is performed on realistic simulated data, which establishes a proof of concept for our framework and defines it as the basis for future extensions.
\end{abstract}

Index Terms-Alternating-direction method of multipliers (ADMM), deconvolution, fluorescence, single-particle reconstruction.

\section{INTRODUCTION}

$\mathbf{F}$ LUORESCENCE microscopy is an essential tool for the observation of subcellular structures and the understanding of their interactions. A large variety of optical devices and reconstruction procedures have been proposed to enhance the resolution of fluorescence images. This research has particularly focused on the lateral plane, where confocal microscopes are finally able to reach the diffraction limit (around $200 \mathrm{~nm}$ ). This barrier is even surpassed by superresolution techniques like structured illumination microscopy (SIM, [1]), stimulated emission depletion (STED, [2]), or photo-activated localization microscopy (PALM) and stochastic optical reconstruction microscopy (STORM) [3]. However, no decisive advance has been realized to improve the axial resolution. As a consequence, the analysis of fluorescence is mainly restricted to $2 \mathrm{D}$ observations, which is likely to loose important information about complex 3D structures.

Manuscript received March 15, 2015; revised July 13, 2015; accepted October 05, 2015. Date of publication October 26, 2015; date of current version January 21,2016 . The guest editor coordinating the review of this manuscript and approving it for publication was Dr. Charles Kervrann.

D. Fortun is with the Signal Processing core of Center for Biomedical Imaging (CIBM-SP), EPFL, 1015 Lausanne, Switzerland (e-mail: denis.fortun@epfl.ch).

P. Guichard is with the Department of Cell Biology, University of Geneva, Sciences III, CH-1211 Geneva, Switzerland.

N. Chu and M. Unser are with the Biomedical Imaging Group, EPFL, 1015 Lausanne, Switzerland.

Color versions of one or more of the figures in this paper are available online at http://ieeexplore.ieee.org.

Digital Object Identifier 10.1109/JSTSP.2015.2493884
To overcome this issue, we propose a novel framework that takes advantage of multiple poses to reconstruct fluorescence images with 3D isotropic high resolution. Our approach is to perform volumetric acquisitions of randomly oriented replicates of a rigid particle. This allows us to take advantage of the high lateral resolution of the microscope to gain access to details within each view. Our reconstruction then combines these details acquired at random poses. This procedure is inspired by the single-particle reconstruction framework used in cryo electron microscopy (cryo-EM) [4], where a particle is reconstructed from tomographic projections instead of volumetric acquisitions. To the best of our knowledge, the only similar existing work for fluorescence is the 3D averaging approach described in [5] for STORM imaging.

Given an input volume, the overall framework is made of three steps: 1) detection and segmentation of individual particles in the original image; 2) estimation of pose parameters; 3 ) $3 \mathrm{D}$ reconstruction. Each of these steps comes with its own challenges that have to be addressed specifically. In this work, we demonstrate the feasibility and the potential of our approach by focusing on the reconstruction problem. Two main challenges have to be overcome: Firstly, the fluorescence imaging model must be adequately integrated in the multiview reconstruction. In particular, the resolution of fluorescence microscopy is usually limited by large point-spread functions (PSF). Secondly, the main difficulty comes from the abundance of input 3D volumes that have to be processed to obtain accurate reconstructions. Such big data require an adapted and efficient algorithm to ensure the computational tractability of the method.

We propose a generic reconstruction framework to overcome the challenges mentioned above. We take the imaging modality into account by designing a joint approach that combines deconvolution and multiview reconstruction in a single model. To cope with the computational issue associated with the high number of views, we adopt a proximal splitting optimization strategy that we adapt to the specific structure of our data. The computational cost of each iteration is independent of the number of input orientations, which is the key point to be able to process large data. Altogether, this allows us to obtain accurate reconstructions with high axial resolution. We demonstrate the efficiency of our method by applying it to a confocal microscopy model. Our work is a proof of concept and a first step toward the realization of a complete and practical framework that would include Steps 1) and 2). Our contributions can be summarized as follows:

- A novel integrative multiview framework for improving the resolution of fluorescence microscopy, and the design 
of a corresponding reconstruction algorithm based on global optimization.

- The capacity to handle a high number of input 3D images at low computational cost, with a proximal splitting optimization strategy which does not depend on the number of views at each iteration.

- A proof of concept establishing the feasibility of our approach in real conditions, based on extensive performance validation on realistic simulated data.

The remainder of the paper is organized as follows: In Section II, we review related works. In Section III, we describe our reconstruction method. In Section IV, we provide an experimental evaluation of the performance of the method. In Section $\mathrm{V}$, we conclude on the main results and discuss the perspectives opened by this work.

\section{RELATED WORK}

\section{A. Averaging in Fluorescence Microscopy}

Recently, the averaging of identical particles in 2D fluorescence imaging has received some particular attention [6], [7]. It has been shown to improve the accuracy of localization in STORM imaging. However, the averaging approach induces a blurring that obliterates the small details of the structures. To partly compensate for this effect, a very large number of particles has to be processed. Moreover, the restriction to 2D prevents one from capturing the whole complexity of 3D shapes, and a strong uncertainty remains on the localization of the proteins under study. In [5], the averaging approach is extended to $3 \mathrm{D}$ for STORM data. The resulting resolution is isotropic in $3 \mathrm{D}$ but suffers from the blurring effect of the averaging.

\section{B. Reconstruction in Cryo-EM}

Cryo-EM measures the electron transmission through particle structures. The images acquired in cryo-EM are thus $2 \mathrm{D}$ projections of the $3 \mathrm{D}$ object, modeled by the Radon transform. The tomographic reconstruction in cryo-EM from observation of instances of a particle at different poses is called single-particle reconstruction (SPR) [8]. It has been a very active research topic over the past thirty years and is today supported by several well established softwares based on a common efficient algorithmic framework (Xmipp [4], Spider [9], Bsoft [10], EMAn [11]).

The similarity of SPR with our framework lies in the idea of reconstructing a particle from several rotated views. However, the image-acquisition model is fundamentally different in our case since we deal with 3D images obtained by convolution with a PSF. Therefore, the reconstruction techniques developed for cryo-EM are not adapted to fluorescence data and dedicated methods have to be designed.

Nevertheless, it is possible to interpret the Radon transform in terms of PSF. Indeed, the Radon transform can be likened to the effect of a PSF that would be made of a line in the axial direction of the microscope, with a width equal to the size of a pixel. Thus, in practice, the Radon transform can be approximated by a very elongated PSF. We shall investigate experimentally the validity of this approximation in Section IV.

\section{Selective-Plane Illumination Microscopy (SPIM)}

The SPIM technique exploits light-sheet illumination to acquire images of a single sample from multiple views [12]. Information at all orientations can be combined in a reconstruction procedure to enhance the resolution [13]-[15]. The concept fundamentally differs from ours since the views are obtained by multiple rotations of a single sample. Thus, the number of views is limited by the photobleaching effect which increases at each new observation. The range of angles that can be covered is also limited by practical constraints on the rotation device. Therefore, existing reconstruction methods [13]-[15] are designed to cope only with a small number of views (five to ten typically). By contrast, we routinely handle several hundreds of views, which creates specific computational issues.

\section{Reconstruction From Multiple Poses}

This section is devoted to the detailed description of our framework and reconstruction method. We first introduce the general framework. Then, we formulate the reconstruction problem. Finally we detail our fast optimization strategy.

\section{A. General Framework}

The underlying assumption of our method is that we are able to acquire a 3D micrograph containing a series of particles replicates that are randomly oriented. The goal is to reconstruct data model of a particle with high isotropic resolution. To this end, three steps have to be performed. The first stage is the identification of individual particles $y_{\boldsymbol{\vartheta}_{i}}$, where $\boldsymbol{\vartheta}_{i}=\left(\boldsymbol{\theta}_{i}, \mathbf{t}_{i}\right)$ is a vector of pose parameters composed by rotation parameters $\boldsymbol{\theta}_{i}$ and translation parameters $\mathbf{t}_{i}$. The second stage is the estimation of the pose parameters $\boldsymbol{\vartheta}_{i}$. The third stage is the reconstruction of the model $x$ of the particle.

Let us assume that the identification step has been performed, providing a set of particles $y_{\vartheta_{i}}$. We consider the case of confocal imaging, for which the image-acquisition process can be modeled as the convolution of the underlying signal with a PSF denoted by $h$. Owing to the small field of view covered by each particle, we assume one spatially invariant PSF per particle. This choice is motivated by the spatial smoothness of the PSF in fluorescence microscopy, which has been experimentally observed in previous works [16], [17]. Each particle can then be modeled as the observation of a rotated and translated version of $x$ obtained at pixel $\mathbf{p}$ by

$$
y_{\boldsymbol{\vartheta}_{i}}(\mathbf{p})=\int_{\mathbb{R}^{3}} h(\mathbf{q}) x\left(\mathbf{R}_{\boldsymbol{\theta}_{i}}(\mathbf{p}-\mathbf{q})+\mathbf{t}_{i}\right) \mathrm{d} \mathbf{q},
$$

where $\mathbf{R}_{\boldsymbol{\theta}_{i}}$ is the rotation matrix with angular parameters $\boldsymbol{\theta}_{i}$. The estimation of the pose $\boldsymbol{\vartheta}_{i}$ allows us to register the observations by applying inverse rotation and translation. It is then possible, after changes of variables, to rewrite the forward model (1) as

$$
y_{\boldsymbol{\vartheta}_{i}}\left(\mathbf{R}_{\boldsymbol{\theta}_{i}}^{-1}\left(\mathbf{p}-\mathbf{t}_{\mathbf{i}}\right)\right)=\int_{\mathbb{R}^{3}} h\left(\mathbf{R}_{\boldsymbol{\theta}_{i}}^{-1} \mathbf{q}\right) x(\mathbf{p}-\mathbf{q}) \mathrm{d} \mathbf{q} .
$$

In a discrete setting, assuming that all images have identical sizes and contain $M$ pixels, we denote $\mathbf{y}_{i} \in \mathbb{R}^{M}$ the observation vector corresponding to the discretization of $y_{\boldsymbol{\vartheta}_{i}}\left(\mathbf{R}_{\boldsymbol{\theta}_{i}}^{-1}\left(\cdot-\mathbf{t}_{\mathbf{i}}\right)\right)$, 
$\mathbf{H}_{\boldsymbol{\vartheta}_{i}} \in \mathbb{R}^{M \times M}$ the convolution matrix representing $h\left(\mathbf{R}_{\boldsymbol{\theta}_{i}}^{-1} \cdot\right)$, and $\mathbf{x} \in \mathbb{R}^{M}$ the unknown image in vector form. The discretization of (2), with the addition of the measurement noise $\mathbf{n} \in \mathbb{R}^{M}$, leads to the linear forward model

$$
\mathbf{y}_{i}=\mathbf{H}_{\boldsymbol{\vartheta}_{i}} \mathbf{x}+\mathbf{n} .
$$

The pose estimation and reconstruction problems are closely intertwined since the degradation model of $\mathbf{x}$ is defined by $\boldsymbol{\vartheta}_{i}$. We formulate the reconstruction problem as a joint maximum a posteriori (MAP) estimation, which can be turned into the optimization problem

$$
\{\widehat{\mathbf{x}}, \widehat{\boldsymbol{\vartheta}}\}=\underset{\mathbf{x} \in \mathbb{R}_{+}^{M}, \boldsymbol{\vartheta} \in \mathbb{R}^{N \times 6}}{\arg \min } \frac{\lambda}{2} f_{\text {data }}(\mathbf{y}, \mathbf{x}, \boldsymbol{\vartheta})+f_{\text {reg }}(\mathbf{x}),
$$

where $\boldsymbol{\vartheta}=\left\{\boldsymbol{\vartheta}_{1}, \ldots, \boldsymbol{\vartheta}_{N}\right\}$ and $\mathbf{y}=\left\{\mathbf{y}_{1}, \ldots, \mathbf{y}_{N}\right\}$ with $N$ the number of particles, the data-fitting term $f_{\text {data }}$ penalizes deviations from the forward model (3), the regularization term $f_{\text {reg }}$ imposes regularity on the solution, and $\lambda>0$ is a tradeoff parameter that balances the two terms. Problem (4) is blind in the sense that the orientations of the PSFs are not known and would need to be estimated jointly with $\mathbf{x}$.

In this proof-of-concept work, we focus on non-blind reconstruction and demonstrate the benefits of our reconstruction method when the pose parameters are known. Thus, we drop the dependency of $f_{\text {data }}$ on $\boldsymbol{\vartheta}$ in the sequel. We focus now on the description of the energy of (4) and its minimization over $\mathbf{x}$.

\section{B. Reconstruction Problem}

The choice of $f_{\text {data }}$ reflects the assumption made on the noise. The image formation in fluorescence microscopy is affected by a combination of two sources of noise. The photo-counting performed by the detector involves a signal-dependent Poisson noise, while thermal and electronic effects produce Gaussian additive noise. For the sake of simplicity and to focus on our proof of concept goal, we neglect the influence of Poisson noise in our model and consider i.i.d. Gaussian noise, which leads to a quadratic data-fitting term of the form

$$
f_{\text {data }}(\mathbf{y}, \mathbf{x})=\sum_{i=1}^{N}\left\|\mathbf{y}_{i}-\mathbf{H}_{\boldsymbol{\vartheta}_{i}} \mathbf{x}\right\|_{2}^{2} .
$$

We discuss the adaptation of our method to Poisson noise in Section IV-E.

The matrix $\mathbf{H}$ is often ill-conditioned. To stabilize the solution, we assume a uniform distribution of the fluorophores in the samples. Then, it is reasonable to favor piecewise constancy of the result. It can be achieved with the total-variation (TV) regularization defined as

$$
f_{\text {reg }}(\mathbf{x})=\|\mathbf{D} \mathbf{x}\|_{1},
$$

where $\mathbf{D}=\left(\mathbf{D}_{x}, \mathbf{D}_{y}, \mathbf{D}_{z}\right)^{\top}$ is a discrete differential operator concatenating first-order forward finite-difference matrices in the three spatial directions.

\section{Minimization}

The main difficulty in the optimization stage stems from the potentially large size of the input data. High accuracy comes at the cost of a large number of orientation angles, as we show in Section IV. Our practical constraint then is to be able to handle this large amount of 3D observations at a reasonable computational cost.

The key element making reconstruction computationally feasible for a very large number of views is our proximal splitting approach, decomposing the original problem into efficiently solvable sub-problems. \{We adopt the alternating-direction method of multipliers (ADMM) [18]-[22], which is adapted to the convex and non-smooth form of (4). Some other related variants based on similar problem splitting could lead to comparable computational savings [23]-[25]. We first reformulate (4) as a constrained optimization problem, by introducing the splitting variables $\mathbf{u}_{1}$ and $\mathbf{u}_{2}$, which leads to

$$
\begin{aligned}
& \min _{\mathbf{x} \in \mathbb{R}^{M}} \frac{\lambda}{2} f_{\text {data }}(\mathbf{y}, \mathbf{x})+\left\|\mathbf{u}_{1}\right\|_{1}+\iota_{\mathbb{R}_{+}^{M}}\left(\mathbf{u}_{2}\right), \\
& \text { s.t. }\left\{\begin{array}{l}
\mathbf{u}_{1}=\mathbf{D} \mathbf{x} \\
\mathbf{u}_{2}=\mathbf{x},
\end{array}\right.
\end{aligned}
$$

where the non-negativity constraint is handled by the indicator function $\iota_{\mathbb{R}_{+}^{M}}$ defined as

$$
\iota_{\mathbb{R}_{+}^{M}}(\mathbf{z})= \begin{cases}0, & \text { if } \mathbf{z} \in \mathbb{R}_{+}^{M} \\ +\infty, & \text { else, }\end{cases}
$$

and where the auxiliary variables $\mathbf{u}_{1}$ and $\mathbf{u}_{2}$ decouple the regularization term and the non-negativity constraint from the data term. We rewrite (7) in the more compact form

$$
\min _{\mathbf{x} \in \mathbb{R}^{N}} \frac{\lambda}{2} f_{\text {data }}(\mathbf{y}, \mathbf{x})+g(\mathbf{u}), \text { s.t. } \mathbf{u}=\mathbf{A} \mathbf{x},
$$

where $\mathbf{u}=\left[\mathbf{u}_{1}, \mathbf{u}_{2}\right]^{\top}, \mathbf{A}=[\mathbf{D}, \mathbf{I}]^{\top}$ with $\mathbf{I}$ the identity matrix, and $g(\mathbf{u})=\left\|\mathbf{u}_{1}\right\|_{1}+\iota_{\mathbb{E}_{+}^{M}}\left(\mathbf{u}_{2}\right)$. The augmented-Lagrangian formulation of the problem (9) is then

$L_{\mu}(\mathbf{x}, \mathbf{u}, \boldsymbol{\alpha})=\frac{\lambda}{2} f_{\text {data }}(\mathbf{x})+g(\mathbf{u})+\boldsymbol{\alpha}^{\top}(\mathbf{A} \mathbf{x}-\mathbf{u})+\frac{\mu}{2}\|\mathbf{A} \mathbf{x}-\mathbf{u}\|_{2}^{2}$,

where $\boldsymbol{\alpha}=\left[\boldsymbol{\alpha}_{1}, \boldsymbol{\alpha}_{2}\right]^{\top}$ is the Lagrange multiplier with $\boldsymbol{\alpha}_{1} \in \mathbb{R}^{3 M}, \boldsymbol{\alpha}_{2} \in \mathbb{R}^{M}$, and $\mu$ is the parameter associated to the quadratic penalty. Problem (4) is then solved by the following alternated updates of the variables:

$$
\begin{aligned}
& \mathbf{x}^{k+1}=\underset{\mathbf{x} \in \mathbb{R}^{M}}{\arg \min } L_{\mu}\left(\mathbf{x}, \mathbf{u}^{k}, \boldsymbol{\alpha}^{k}\right) \\
& \mathbf{u}^{k+1}=\underset{\mathbf{u} \in \mathbb{R}^{M}}{\arg \min } L_{\mu}\left(\mathbf{x}^{k+1}, \mathbf{u}, \boldsymbol{\alpha}^{k}\right) \\
& \boldsymbol{\alpha}^{k+1}=\boldsymbol{\alpha}^{k}+\mu\left(\mathbf{A} \mathbf{x}^{k+1}-\mathbf{u}^{k+1}\right) .
\end{aligned}
$$

The two components of $\mathbf{u}$ are independent in (12), so that the minimization w.r.t. the two variables can be performed separately. The update of $\mathbf{u}_{1}$ amounts to the computation of the proximal operator of the $\ell_{1}$ norm, which has a unique solution that can easily be computed by soft-thresholding operations [26] like

$$
\mathbf{u}_{1}^{k+1}=\max \left(\left\|\mathbf{v}_{1}^{k}\right\|_{1}-\frac{1}{\mu}, 0\right) \frac{\mathbf{v}_{1}^{k}}{\left\|\mathbf{v}_{1}^{k}\right\|_{1}},
$$

with $\mathbf{v}_{1}^{k}=\mathbf{D} \mathbf{x}^{k+1}+(1 / \mu) \boldsymbol{\alpha}_{1}^{k}$.

The update of $\mathbf{u}_{2}$ is the projection onto the set $\mathbb{R}_{+}^{M}$

$$
\mathbf{u}_{2}^{k+1}=\max \left(\mathbf{v}_{2}^{k}, 0\right),
$$

with $\mathbf{v}_{2}^{k}=\mathbf{x}^{k+1}-(1 / \mu) \boldsymbol{\alpha}_{2}^{k}$. 
For $\mathbf{u}^{k}$ fixed, (11) is a pixelwise quadratic problem solvable in closed form as

$$
\begin{aligned}
\mathbf{x}^{k+1}= & \left(\mu \mathbf{A}^{\top} \mathbf{A}+\lambda \sum_{i=1}^{N} \mathbf{H}_{\boldsymbol{\vartheta}_{i}^{\top}}^{\top} \mathbf{H}_{\boldsymbol{\vartheta}_{i}}\right)^{-1} \\
& \times\left(\mu \mathbf{A}^{\top}\left(\mathbf{u}^{k}-\frac{\boldsymbol{\alpha}^{k}}{\mu}\right)+\lambda \sum_{i=1}^{N} \mathbf{H}_{\boldsymbol{\vartheta}_{i}^{\top}}^{\top} \mathbf{y}_{i}\right) .
\end{aligned}
$$

Most of the computational cost of our method is devoted to the filtering operations involving $\mathbf{H}_{\boldsymbol{\vartheta}_{i}}^{\top}$ in (16). The bulk of this cost comes from the summation over the number $N$ of particles, which can be large. The crucial advantage of the ADMM approach is to decouple the $N$ filterings by $\mathbf{H}_{\boldsymbol{\vartheta}}^{\top}$ from the iterative updates. The sums can then be computed offline, which makes the cost of each iteration independent of the number of orientations.

Another computational issue comes from the fact that the matrix $\left(\mu \mathbf{A}^{\top} \mathbf{A}+\lambda \sum_{i=1}^{N} \mathbf{H}_{\boldsymbol{\vartheta}_{i}}^{\top} \mathbf{H}_{\boldsymbol{\vartheta}_{i}}\right)$ is too large to be inverted. However, due to the block-circulant form of the matrices $\mathbf{H}_{\boldsymbol{\vartheta}_{i}}$ and $\mathbf{D}$, they can be diagonalized by the discrete Fourier transform under periodic boundary conditions. After diagonalization, the update (16) can be evaluated much faster in the Fourier domain as

$$
\mathbf{x}^{k+1} \mathcal{F}^{-1}\left\{\frac{\mathcal{F}\left\{\mu \mathbf{A}^{\top}\left(\mathbf{u}^{k}-\frac{\boldsymbol{\alpha}^{k}}{\mu}\right)\right\}+\lambda \sum_{i=1}^{N} \mathcal{F}\left\{\mathbf{H}_{\boldsymbol{\vartheta}_{i}}^{\top} \mathbf{y}_{i}\right\}}{\mu\left(|\mathcal{F}\{\mathbf{D}\}|^{2}+\mathbf{I}\right)+\lambda \sum_{i=1}^{N}\left|\mathcal{F}\left\{\mathbf{H}_{\boldsymbol{\vartheta}_{i}}\right\}\right|^{2}}\right\} .
$$

The terms $\sum_{i=1}^{N} \mathcal{F}\left\{\mathbf{H}_{\boldsymbol{\vartheta}_{i}}^{\top} \mathbf{y}_{i}\right\}$ and $\mu\left(|\mathcal{F}\{\mathbf{D}\}|^{2}+\mathbf{I}\right)+$ $\lambda \sum_{i=1}^{N}\left|\mathcal{F}\left\{\mathbf{H}_{\boldsymbol{\vartheta}_{i}}\right\}\right|^{2}$ are constant and are thus computed offline. The computational time of each iteration is then mostly devoted to two FFTs, and the total number of FFT computations to reconstruct a particle is $2\left(N+n_{\text {it }}+1\right)$, where $n_{\text {it }}$ is the number of iterations of the ADMM optimization. The time complexity of the algorithm is thus $\mathcal{O}(N \log (N))$.

Finally, the choice of the parameter $\mu$ does not influence much the final result, but has a significant influence on the speed of convergence. A large value of $\mu$ accelerates convergence but favors local optima. Based on empirical observations, we follow the continuation strategy of [18] by updating the value of $\mu$ at each iteration as described in Algorithm 1. The constants $\beta$ and $\gamma$ have been determined experimentally to optimize the speed of convergence. This approach yields faster convergence and similar results than a fixed value of $\mu$. The steps of the overall algorithm are recalled and summarized in Algorithm 1.

\section{RESULTS}

\section{A. Experimental Protocol}

We exploit the public database EMDataBank ${ }^{1}$, which is the reference portal for deposit, retrieval, and evaluation of $3 \mathrm{D}$ density maps in cryo-EM. The reconstructed volumes in cryo-EM can attain subnanometric resolution, while typical resolutions in fluorescence imaging are comprised between $200 \mathrm{~nm}$ in confocal and $30 \mathrm{~nm}$ in PALM/STORM imaging. Therefore, we considered cryo-EM reconstructions as the ground truth to evaluate the performance of our reconstruction for fluorescence imaging.

\section{Algorithm 1 Joint reconstruction and deconvolution}

$$
\begin{aligned}
& \text { Input: } \mathbf{y}_{i}, \mathbf{H}_{\boldsymbol{\vartheta}_{i}}, \lambda, \mu \\
& \text { Initialization: } \mathbf{x}^{0}=\mathbf{y}, \mathbf{u}_{1}=\mathbf{0}, \mathbf{u}_{2}=\mathbf{0}, \boldsymbol{\alpha}_{1}=\mathbf{0}, \boldsymbol{\alpha}_{2}=\mathbf{0} \\
& \text { Constants: } \beta=0.6, \gamma=2 \\
& \mathbf{C}_{1} \leftarrow \lambda \sum_{i=1}^{N} \mathcal{F}\left\{\mathbf{H}_{\boldsymbol{\vartheta}_{i}}^{\top} \mathbf{y}_{i}\right\} \\
& \mathbf{C}_{2} \leftarrow \mu\left(|\mathcal{F}\{\mathbf{D}\}|^{2}+\mathbf{I}\right)+\lambda \sum_{i=1}^{N}\left|\mathcal{F}\left\{\mathbf{H}_{\boldsymbol{\vartheta}_{i}}\right\}\right|^{2} \\
& \text { while stopping criterion do } \\
& \mathbf{x}^{k+1}=\mathcal{F}^{-1}\left\{\left(\mathcal{F}\left\{\mu \mathbf{A}^{\top}\left(\mathbf{u}^{k}-\boldsymbol{\alpha}^{k} / \mu\right)\right\}+\mathbf{C}_{1}\right) / \mathbf{C}_{2}\right\} \\
& \mathbf{v}_{1}^{k}=\mathbf{D} \mathbf{x}^{k+1}+(1 / \mu) \boldsymbol{\alpha}_{1}^{k} \\
& \mathbf{v}_{2}^{k}=\mathbf{x}^{k+1}-(1 / \mu) \boldsymbol{\alpha}_{2}^{k} \\
& \mathbf{u}_{1}^{k+1}=\max \left(\left\|\mathbf{v}_{1}^{k}\right\|_{1}-1 / \mu, 0\right)\left(\mathbf{v}_{1}^{k} /\left\|\mathbf{v}_{1}^{k}\right\|_{1}\right) \\
& \mathbf{u}_{2}^{k+1}=\max \left(\mathbf{v}_{2}^{k}, 0\right) \\
& \boldsymbol{\alpha}_{1}^{k+1}=\boldsymbol{\alpha}_{1}^{k}+\mu\left(\mathbf{D} \mathbf{x}^{k+1}-\mathbf{u}_{1}^{k+1}\right) \\
& \boldsymbol{\alpha}_{2}^{k+1}=\boldsymbol{\alpha}_{2}^{k}+\mu\left(\mathbf{x}^{k+1}-\mathbf{u}_{2}^{k+1}\right) \\
& \text { if }\left\|\mathbf{A} \mathbf{x}^{k+1}-\mathbf{u}^{k+1}\right\|_{2}<\beta\left\|\mathbf{A} \mathbf{x}^{k}-\mathbf{u}^{k}\right\|_{2} \\
& \mu \leftarrow \mu \times \gamma \\
& \text { end } \\
& k \leftarrow k+1
\end{aligned}
$$

To circumvent the absence of existing work realizing Steps 1 and 2 of the SPR framework for fluorescence (defined in Section I as particle detection and orientation estimation), we generate input data from the reconstructions of the EMDataBank. Our working assumption is that the structure of the particles can be imaged by fluorescent tagging, with a uniform distribution of fluorophores. Hence, our approach to generated realistic 3D fluorescence images is to rotate the cryo-EM reconstructions, threshold the density map, apply PSF convolution, and add Gaussian noise. The orientation angles are assigned by random sampling on the unit sphere. The resulting fluorescence images of rotated particles are used as input data for the reconstruction method. Examples can be visualized in Fig. 3(c)-(e).

In the EMDataBank database, we selected the nuclear pore reconstruction described in [28], the flagellar basal body of [29], and the centriole basal body presented in [27]. These particles are characterized by their large size, enabling imaging by confocal microscopy. The three reconstructions are rendered in Fig. 1. Protein mapping in the centriole structure has recently received particular attention and motivated several works in superresolution [30]-[32].

\section{B. Comparison of Methods}

The only existing competing method for reconstruction from multiple poses in fluorescence is described in [5]. It is an averaging of the registered particles. We refer to this approach as Average in the sequel. For a fair evaluation of our approach, we also implemented two other reconstruction methods.

\footnotetext{
${ }^{1}$ http://www.emdatabank.org/search.html
} 
TABLE I

Comparison of PSNR (DB) Between JoInt, DeConV/AVERaGe, Tomo [33] and AVERAGE [5], FOR 100 ORIENTATION ANGLES, SEVERAL LEVELS OF NOISE AND PSF SizES



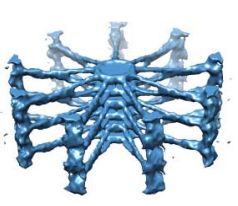

Centriole [27]

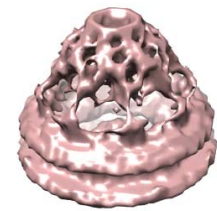

Nuclear pore [28]

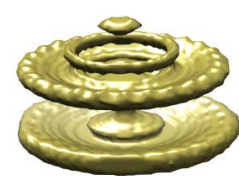

Flagella basal body [29]
Fig. 1. 3D surface visualization of the three ground truth particles. The input particle images have dimensions from $50 \times 50 \times 20$ to $70 \times 70 \times 35$ pixels.

We first consider applying to fluorescence the standard multiview reconstruction from projections modeled by the Radon transform. We use the tomographic reconstruction method described in [33], which embeds the Radon transform in a TV regularization and ADMM optimization strategy that is similar to ours. We refer to this method as Tomo in the sequel.

We also implemented an extension of [5] as follows: 1) We first independently deconvolved each volume of rotated particle, 2 ) the particles were then registered (the registration was exact since we know the poses), 3) the registered particles were averaged. Compared to [5], the deconvolution step is crucial because it depends on the PSF. For the sake of fairness, we implemented a deconvolution method in the same framework as ours, with TV regularization and ADMM optimization. We call this deconvolution/averaging method as Deconv/Average in the sequel, and we name our joint deconvolution and reconstruction Joint.

\section{Implementation Details}

The algorithms were implemented in Matlab and the experiments have been carried out on a PC with $2.60 \mathrm{GHz}$ CPU and 128 GB RAM. The regularization parameters yielding the best PSNR have been selected for the methods Tomo, Deconv/Average, and Joint in all experiments, after extensive tests for a large range of values. The stopping criterion of the optimization process for these methods was fixed to $10^{-5}$ to guarantee convergence. All input images are nonnegative and scaled to have

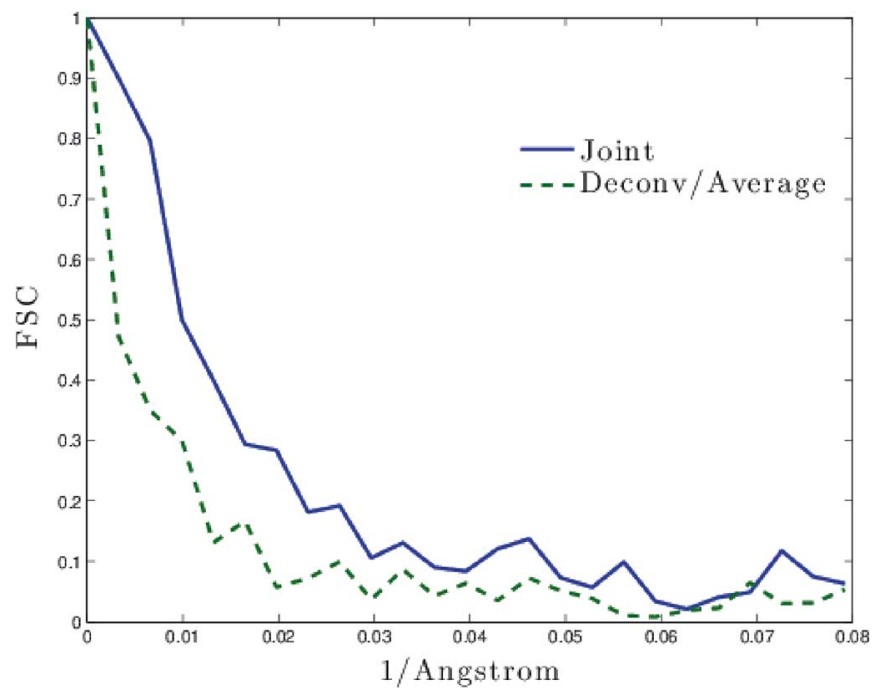

Fig. 2. Comparison of Fourier shell correlation curves of Joint and Deconv/ Average.

their maximum at 255. The PSF are generated according to the Born\&Wolf model with the available PSF Generator software ${ }^{2}$ described in [34].

\section{Results}

The quality of the reconstruction is measured by the peak signal-to-noise ratio (PSNR) defined by $10 \log _{10}\left(L^{2} / M S E\right)$, where $L$ is the maximum intensity of the image and $M S E$ is the mean-square error defined by $M S E=\left\|\widehat{\mathbf{x}}-\mathbf{x}_{0}\right\|^{2} / N$, with $x_{0}$ the ground truth. We also evaluate our results with the Fouriershell correlation (FSC), which is the standard tool for measuring the resolution of reconstructed volumes. It correlates the Fourier

\footnotetext{
${ }^{2} \mathrm{http} / / /$ bigwww.epfl.ch/algorithms/psfgenerator/
} 


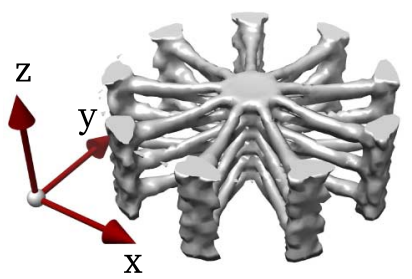

(a) Ground truth

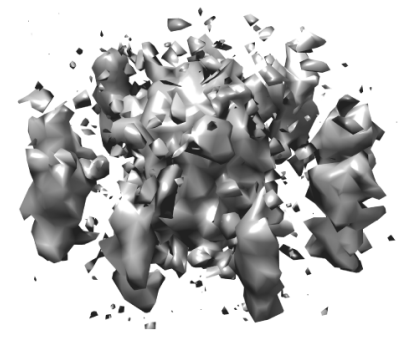

(c) Example 1 of Input particle

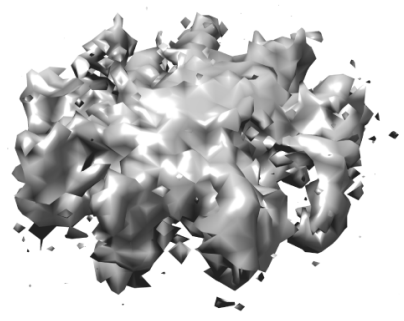

(e) Example 2 of Input particle
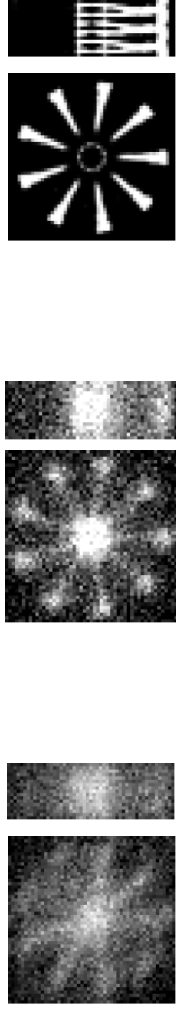


(b) Joint reconstruction
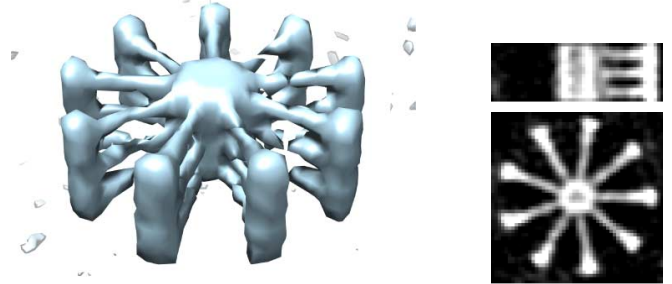

(d) Deconv/Average reconstruction

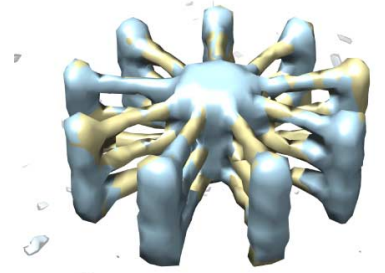

(f) Joint + Deconv/Average reconstruction

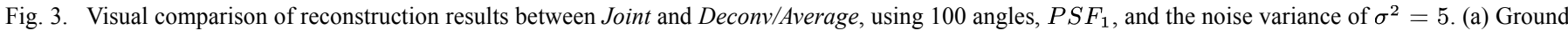

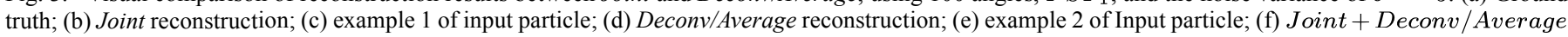
reconstruction.

transforms of two estimations $\widehat{\mathbf{x}}_{1}$ and $\widehat{\mathbf{x}}_{2}$ obtained with different input data at different frequency ranges and is defined as

$$
F S C(r)=\frac{\sum_{r_{i} \in r} \mathcal{F}\left(\widehat{\mathbf{x}}_{1}\right)\left(r_{i}\right) \mathcal{F}\left(\widehat{\mathbf{x}}_{2}\right)^{*}\left(r_{i}\right)}{\sqrt{\sum_{r_{i} \in r}\left|\mathcal{F}\left(\widehat{\mathbf{x}}_{1}\right)\left(r_{i}\right)\right|^{2} \sum_{r_{i} \in r}\left|\mathcal{F}\left(\widehat{\mathbf{x}}_{2}\right)^{*}\left(r_{i}\right)\right|^{2}}} .
$$

The resolution is defined as the frequency $r_{0}$ at which $F S C\left(r_{0}\right)=0.5$.

Influence of Noise and PSF Size: We report in Table I the PSNR of the reconstructions obtained with Joint, Deconv/Average, Average, and Tomo, obtained for 100 angles. The experiments were realized with several levels of noise, and with PSF sizes defined in pixels as follows: $(69 \times 69 \times 43)$ for $\mathrm{PSF}_{1}$, $(35 \times 35 \times 21)$ for $\mathrm{PSF}_{2}$, and $(17 \times 17 \times 11)$ for $\mathrm{PSF}_{3}$. The input volume sizes vary from $(50 \times 50 \times 20)$ to $(70 \times 70 \times 35)$. Note that the most realistic PSF is $\mathrm{PSF}_{1}$, which is the largest one.

It can be first noticed that the results of Tomo and Average in Table I are clearly far from being competitive with the other two methods. Because of these poor results, we focus the remainder of the experimental analysis on Joint and Deconv/Average.

The results obtained with Joint are superior to those of Deconv/Average in almost all cases of Table I. In particular, Joint outperforms Deconv/Average for all levels of noise. We notice that the gain in performance of Joint over Deconv/Average increases with the size of the PSF. This is an important observation since the most realistic PSF is the largest one.
Resolution Improvement: In Fig. 2, we plot the FSC of Joint and Deconv/Average for the case of the centriole. The curve of Joint is clearly above the one of Deconv/Average, meaning that its similarity with the ground truth is higher at all frequencies. As a result, we achieve a significantly finer resolution of $101 \mathrm{~nm}$, whereas the resolution of Deconv/Average is $333 \mathrm{~nm}$.

Visual Results: Reconstructions obtained with Joint and Deconv/Average can be visualized in Fig. 3 for the case of the centriole. 3D surface renderings are obtained with the same threshold value in Figs. 3(a), (b), (d), (f). The 2D images of the central $x-y$ and $y-z$ slices are also displayed in each case. The amount of blur and noise of the input volumes can be appreciated in Figs. 3(c)-(e) for two different rotation angles. The details of the structure are better preserved by Joint, especially at the thin spokes of the structure, which are often discarded by Deconv/Average. The superimposition of the results of Joint and Deconv/Average in Fig. 3(f) makes also appear the deformation of the structure. In particular, the central tube and the pinheads at the borders of the centriole are oversmoothed and appear larger in the Deconv/Average reconstruction.

Influence of the Number of Poses: The influence of the number of poses on the PSNR of the reconstructions of Joint and Deconv/Average is reported in Fig. 4. Several levels of noise are considered. Increasing the number of poses improves the accuracy of both methods. However, the PSNR of Joint grows significantly faster than the one of Deconv/Average, for all levels of noise. 


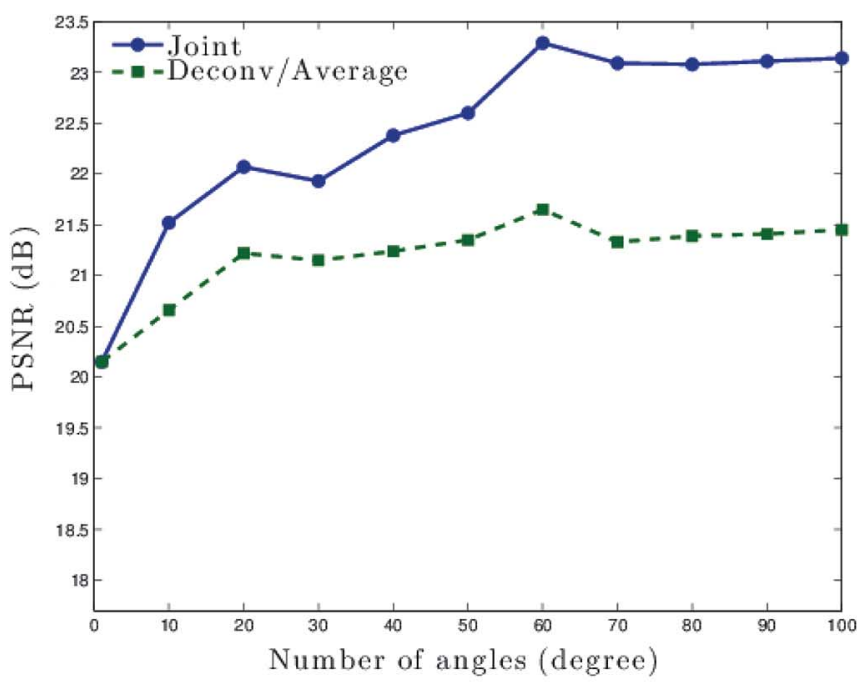

variance $=0.5$

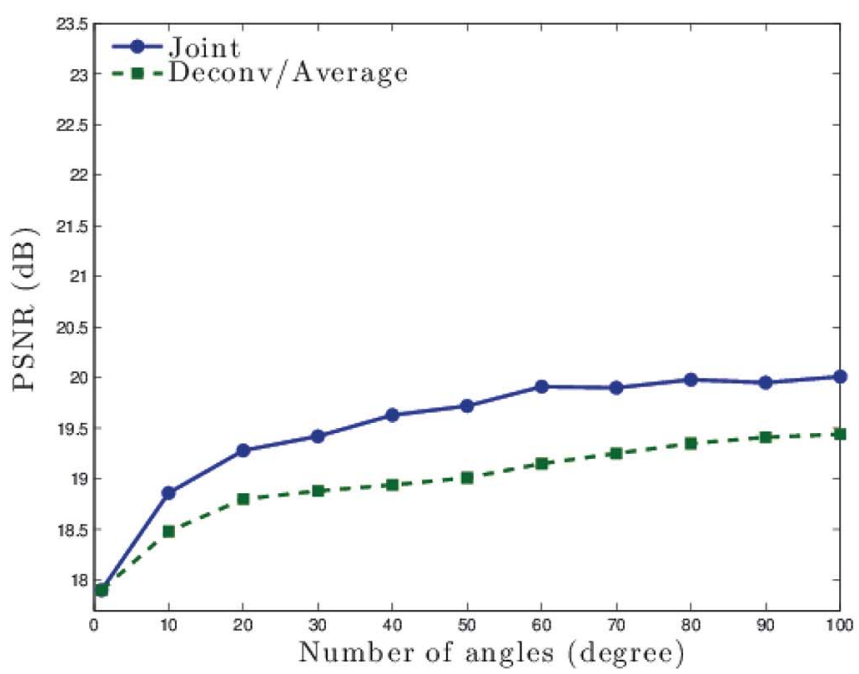

variance $=10$

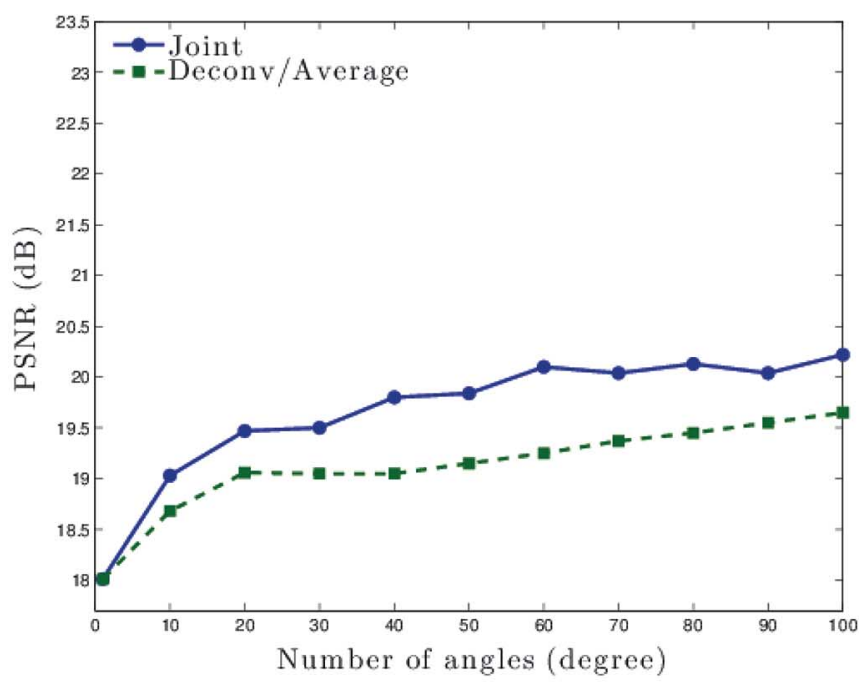

variance $=5$

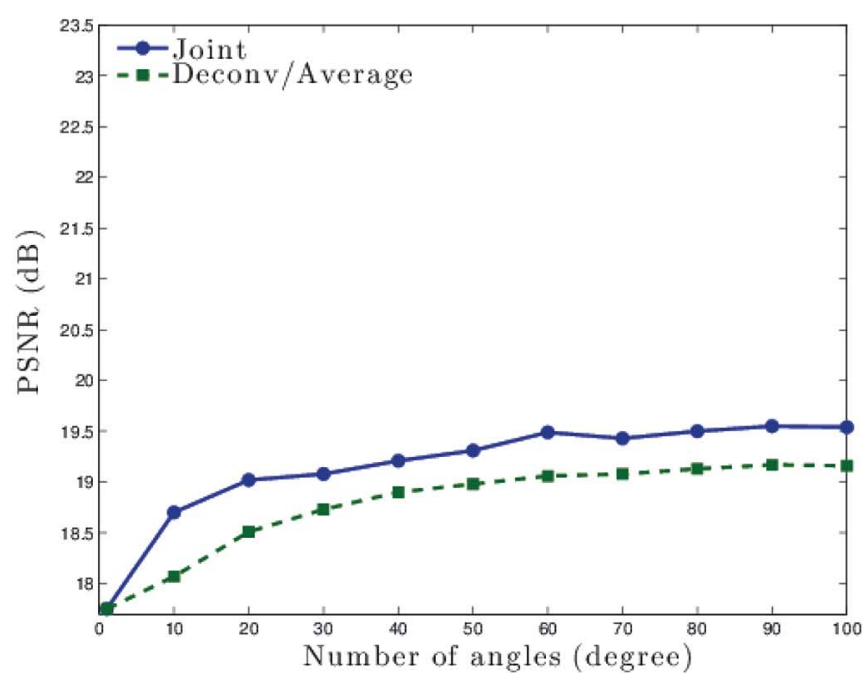

variance $=15$

Fig. 4. Influence of the number of orientations for Joint and Deconv/Average in the case of the centriole and using $\mathrm{PSF}_{1}$.

Computational Time: Finally, the computational times of Joint and Deconv/Average are compared in Fig. 5 in the case of the centriole and for $\mathrm{PSF}_{1}$. We consider the same numbers of poses as in Fig. 4, to be able to compare the evolutions of the computational time and the PSNR. We observe that the computational time of Joint stays almost constant as the number of poses increases, whereas it grows much faster in the case of Deconv/Average. For 100 poses, Joint takes $30 \mathrm{~s}$ and Deconv/Average takes $29 \mathrm{~min}$.

\section{E. Discussion}

We point out in the sequel the main conclusions that can be deduced from the experimental results described above, demonstrating the feasibility of our approach.

The poor results of Tomo invalidate the approximation of the PSF as a line made by the Radon transform. The tomographic approach, which is the standard model for most multiview reconstruction problems is thus insufficient in our case. This result demonstrates the necessity of a model dedicated to fluorescence imaging.



Fig. 5. Impact of the number of orientations on the computational time of Joint and Deconv/Average. 
The low accuracy of Average, and the gap separating it from Deconv/Average (which differs from Average only by a deconvolution step), emphasizes the importance of taking into account the PSF in the reconstruction procedure. However, the independent deconvolutions performed by Deconv/Average are not the proper way to integrate the PSF in the reconstruction. Indeed, as can be seen in Fig. 3(c)-(e), the poor axial resolution induces a particularly large blur in the axial direction of the PSF, discarding almost all information along that axis. As a consequence, the independent deconvolutions performed by Deconv/Average are inefficient and the averaging is affected by these errors. Our approach is less sensitive to this effect since the joint model for deconvolution and reconstruction avoids the drawback of separate deconvolution and averaging. This explains the superiority of Joint over Deconv/Average in Table I.

In addition to these quantitative improvements, our method is particularly well adapted to practical constraints that can be encountered in real conditions. Firstly, the robustness of the joint reconstruction to large PSF sizes is crucial since low resolution is the main limitation of confocal imaging. Secondly, the fast increase of the PSNR with the number of poses reported in Fig. 4 is very important in practice because it allows us to reduce the required number of particles and can facilitate the preparation of samples for the biologists.

The low computational cost of our reconstruction algorithm is a very desirable feature that makes the integration of a large number of particles feasible. Indeed, dealing with a large number of 3D input images leads to a prohibitive computational cost in the case of Deconv/Average, a drawback which our proposed approach overcomes. Moreover, it is also important to keep in mind that in practice the reconstruction process needs to be performed several times, alternatively with angles estimation, increasing even more the computational cost. The iterative part of our method, which contains most of the computational cost, does not depend on the number of poses. More precisely, the computational cost of Joint is mainly devoted to the computation of $2\left(N+n_{\mathrm{it}}+1\right)$ FFT. In comparison, Deconv/Average repeats the deconvolution process for each angle and thus requires $2 N\left(N+n_{\text {it }}+1\right)$ FFT computations. Consequently, the computational complexity of Joint is $\mathcal{O}(N$ $\log (N))$, while Deconv/Average is in $\mathcal{O}\left(N^{2} \log (N)\right)$. The ability to process many different orientations is crucial for the accuracy of the result, as emphasized in Fig. 4, and made possible by our approach.

A natural extension of our method would be to take into account the Poisson noise usually involved in fluorescence microscopy. It would require to redefine the data term (5) with the Kullback-Leibler divergence [35]-[37]. In this case, our minimization framework described in Section III-C could be easily adapted, similarly to previous works [35]-[37]. More precisely, an additional splitting variable should be introduced in the constrained formulation (7) to split the data term. The proximity operator of the Kullback-Leibler divergence would then have to be computed, which can be done in analytical form. The other steps of Algorithm 1 would remain similar, and the independence of the computational cost from the number of views would be retained.
Another problem that remains to be addressed is the estimation of the pose of each particle. While it constitutes a research project on its own right, it can benefit from some previous work in other fields. In particular, two strategies can be envisaged. A first option is to register all the particles on a common reference. This approach can take advantage of the high quality of rigid-body registration achieved in medical imaging [38]. Alternatively, one may take inspiration from the methodology for pose estimation from projections which has been perfected over years in cryo-EM tomography [39] and which may be adapted to the present forward model. Therefore, despite the difficulty of the problem, the good results and mature development of these two fields suggest the feasibility of pose estimation in our case.

\section{CONCLusion}

We have proposed a novel approach for reconstructing fluorescence volumes with isotropic high resolution. We exploit multiple poses of particle replicates to combine multiview reconstruction and deconvolution in the same model. The computational cost of the iterative part of our optimization approach does not depend on the number of input volumes. This allows us to tackle the challenge of processing a large amount of 3D data in low computational time. Experimental results demonstrate the feasibility of our scheme by achieving accurate reconstructions, as well as proving robustness to practical constraints as large PSF sizes and large number of poses. Our method also outperforms similar approaches based on averaging in all situations.

\section{REFERENCES}

[1] M. G. L. Gustafsson, "Surpassing the lateral resolution limit by a factor of two using structured illumination microscopy," J. Microscopy, vol. 198 , no. 2, pp. 82-87, 2000.

[2] H. W. and J. Wichmann, "Breaking the diffraction resolution limit by stimulated emission: Stimulated-emission-depletion fluorescence microscopy," Opt. Lett., vol. 19, no. 11, pp. 780-782, 1994.

[3] E. Betzig, G. H. Patterson, R. Sougrat, O. W. Lindwasser, S. Olenych, J. S. Bonifacino, M. W. Davidson, J. Lippincott-Schwartz, and H. F. Hess, "Imaging intracellular fluorescent proteins at nanometer resolution," Science, vol. 313, no. 5793, pp. 1642-1645, 2006.

[4] S. H. W. Scheres, R. Núñez-Ramírez, C. Ó. S. Sorzano, J. M. Carazo, and R. Marabini, "Image processing for electron microscopy singleparticle analysis using Xmipp," Nature Protocols, vol. 3, no. 6, pp. 977-990, 2008.

[5] J. Broeken, H. Johnson, D. S. Lidke, S. Liu, R. P. J. Nieuwenhuizen, S. Stallinga, K. A. Lidke, and B. Rieger, "Resolution improvement by 3D particle averaging in localization microscopy," Meth. Applicat. Fluoresc., vol. 3, no. 1, p. 014003, 2015.

[6] A. Lóschberger, S. van de Linde, M. C. Dabauvalle, B. Rieger, M. Heilemann, G. Krohne, and M. Sauer, "Super-resolution imaging visualizes the eightfold symmetry of gp210 proteins around the nuclear pore complex and resolves the central channel with nanometer resolution," J. Cell Sci., vol. 125, no. 3, pp. 570-575, 2012.

[7] A. Szymborska, A. de Marco, N. Daigle, V. C. Cordes, J. A. G. Briggs and J. Ellenberg, "Nuclear pore scaffold structure analyzed by superresolution microscopy and particle averaging," Science, vol. 341, no. 6146, pp. 655-658, 2013.

[8] A. F. Fercher, W. Drexler, C. K. Hitzenberger, and T. Lasser, "Optical coherence tomography-principles and applications," Rep. Progr. Phys., vol. 66 , no. 2, p. 239, 2003

[9] T. R. Shaikh, H. Gao, W. T. Baxter, F. J. Asturias, N. Boisset, A. Leith, and J. Frank, "Spider image processing for single-particle reconstruction of biological macromolecules from electron micrographs," Nature Protocols, vol. 3, no. 12, pp. 1941-1974, 2008. 
[10] J. B. Heymann, G. Cardone, D. C. Winkler, and A. C. Steven, "Computational resources for cryo-electron tomography in Bsoft," J. Struct. Biol., vol. 161, no. 3, pp. 232-242, 2008.

[11] G. Tang, L. Peng, P. R. Baldwin, D. S. Mann, W. Jiang, I. Rees, and S. J. Ludtke, "EMAN2: An extensible image processing suite for electron microscopy," J. Struct. Biol., vol. 157, no. 1, pp. 38-46, 2007.

[12] J. Huisken, J. Swoger, F. Del Bene, J. Wittbrodt, and E. H. K. Stelzer, "Optical sectioning deep inside live embryos by selective plane illumination microscopy," Science, vol. 305, no. 5686, pp. 1007-1009, 2004.

[13] U. Krzic, "Multiple-view microscopy with light-sheet based fluorescence microscope," Ph.D. dissertation, Heidelberg Univ., Heidelberg, Germany, 2009.

[14] M. Temerinac-Ott, O. Ronneberger, P. Ochs, W. Driever, T. Brox, and H. Burkhardt, "Multiview deblurring for 3-D images from light-sheetbased fluorescence microscopy," IEEE Trans. Image Process., vol. 21, no. 4, pp. 1863-1873, 2012.

[15] S. Preibisch, F. Amat, E. Stamataki, M. Sarov, R. H. Singer, E. Myers, and P. Tomancak, "Efficient Bayesian-based multiview deconvolution," Nature Meth., vol. 11, no. 6, pp. 645-648, 2014.

[16] C. Preza and J. A. Conchello, "Depth-variant maximum-likelihood restoration for three-dimensional fluorescence microscopy," JOSA A, vol. 21, no. 9, pp. 1593-1601, 2004.

[17] S. Ben Hadj, L. Blanc-Féraud, G. Aubert, and G. Engler, "Blind restoration of confocal microscopy images in presence of a depth-variant blur and Poisson noise," in Proc. IEEE Int. Conf. Acoust., Speech, Signal Process., 2013, pp. 915-919.

[18] Y. Wang, J. Yang, W. Yin, and Y. Zhang, "A new alternating minimization algorithm for total variation image reconstruction," SIAM J. Imag. Sci., vol. 1, no. 3, pp. 248-272, 2008.

[19] M. Tao and J. Yang, "Alternating direction algorithms for total variation deconvolution in image reconstruction," Dept. of Math., Nanjing Univ., Nanjing, China, TR0918, 2009.

[20] S. Boyd, N. Parikh, E. Chu, B. Peleato, and J. Eckstein, "Distributed optimization and statistical learning via the alternating direction method of multipliers," Foundat. Trends Mach. Learn., vol. 3, no. 1, pp. 1-122, 2011.

[21] M. V. Afonso, J. M. Bioucas-Dias, and M. Figueiredo, “An augmented Lagrangian approach to the constrained optimization formulation of imaging inverse problems," IEEE Trans. Image Process., vol. 20, no. 3, pp. 681-695, Mar. 2011.

[22] S. Ramani and J. A. Fessler, "A splitting-based iterative algorithm for accelerated statistical x-ray CT reconstruction," IEEE Trans. Med. Imag., vol. 31, no. 3, pp. 677-688, Mar. 2012.

[23] P. L. Combettes and J. C. Pesquet, "Proximal splitting methods in signal processing," in Fixed-Point Algorithms for Inverse Problems in Science and Engineering. New York, NY, USA: Springer, 2011, pp. $185-212$.

[24] A. Chambolle and T. Pock, "A first-order primal-dual algorithm for convex problems with applications to imaging," J. Math. Imag. Vis. vol. 40, no. 1, pp. 120-145, 2011.

[25] N. Pustelnik, C. Chaux, and J. C. Pesquet, "Parallel proximal algorithm for image restoration using hybrid regularization," IEEE Trans. Image Process., vol. 20, no. 9, pp. 2450-2462, Sep. 2011.

[26] P. Combettes and V. Wajs, "Signal recovery by proximal forward-backward splitting," Multiscale Model. Simulat., vol. 4, no. 4, pp. 1168-1200, 2005.

[27] P. Guichard, V. Hachet, N. Majubu, A. Neves, D. Demurtas, N. Olieric, I. Fluckiger, A. Yamada, K. Kihara, Y. Nishida, S. Moriya, M. O. Steinmetz, Y. Hongoh, and P. Gönczy, "Native architecture of the centriole proximal region reveals features underlying its 9-fold radial symmetry," Current Biol., vol. 23, no. 17, pp. 1620-1628, 2013.

[28] M. Beck, F. Förster, M. Ecke, J. M. Plitzko, F. Melchior, G. Gerisch, W. Baumeister, and O. Medalia, "Nuclear pore complex structure and dynamics revealed by cryoelectron tomography," Science, vol. 306, no. 5700, pp. 1387-1390, 2004

[29] A. Kawamoto, Y. V. Morimoto, T. Miyata, T. Minamino, K. T. Hughes, T. Kato, and K. Namba, "Common and distinct structural features of salmonella injectisome and flagellar basal body," Sci. Rep., vol. 3, 2013

[30] K. F. Sonnen, L. Schermelleh, H. Leonhardt, and E. A. Nigg, "3Dstructured illumination microscopy provides novel insight into architecture of human centrosomes," Biol. Open, vol. 1, no. 10, pp. 965-976, 2012.
[31] L. Lau, Y. L. Lee, S. J. Sahl, T. Stearns, and W. E. Moerner, "STED microscopy with optimized labeling density reveals 9 -fold arrangement of a centriole protein," Biophys. J., vol. 102, no. 12, pp. 2926-2935, 2012.

[32] D. Keller, M. Orpinell, N. Olivier, M. Wachsmuth, R. Mahen, R. Wyss, V. Hachet, J. Ellenberg, S. Manley, and P. Gonczy, "Mechanisms of HsSAS-6 assembly promoting centriole formation in human cells," $J$. Cell Biol., vol. 204, no. 5, pp. 697-712, 2014.

[33] M. Nilchian, C. Vonesch, P. Modregger, M. Stampanoni, and M. Unser, "Fast iterative reconstruction of differential phase contrast x-ray tomograms," Opt. Exp., vol. 21, no. 5, pp. 5511-5528, Mar. 11, 2013.

[34] H. Kirshner, F. Aguet, D. Sage, and M. Unser, "3-D PSF fitting for fluorescence microscopy: Implementation and localization application," J. Microscopy, vol. 249, no. 1, pp. 13-25, 2013.

[35] M. A. T. Figueiredo and J. M. Bioucas-Dias, "Restoration of Poissonian images using alternating direction optimization," IEEE Trans. Image Process., vol. 19, no. 12, pp. 3133-3145, Dec. 2010.

[36] S. Lefkimmiatis, J. P. Ward, and M. Unser, "Hessian Schatten-norm regularization for linear inverse problems," IEEE Trans. Image Process., vol. 22, no. 5, pp. 1873-1888, May 2013.

[37] J. Boulanger, N. Pustelnik, and L. Condat, "Non-smooth convex optimization for an efficient reconstruction in structured illumination microscopy," in Proc. Int. Symp. Biomed. Imag., Beijing, China, Mar. 2014, pp. 995-998.

[38] P. Thévenaz and M. Unser, "Optimization of mutual information for multiresolution image registration," IEEE Trans. Image Process., vol. 9 , no. 12 , pp. 2083-2099, Dec. 2000.

[39] J. Vargas, A. L. Álvarez-Cabrera, R. Marabini, J. M. Carazo, and C. O. S. Sorzano, "Efficient initial volume determination from electron microscopy images of single particles," Bioinformatics, vol. 30, no. 20, pp. 2891-2898, 2014.

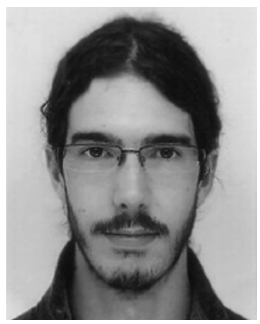

Denis Fortun graduated from Télécom Physique Strasbourg and received the M.Sc. degree in engineering and image processing from the University of Strasbourg, France, in 2010, and the Ph.D. degree in signal analysis and telecommunication in 2014 from the University of Rennes 1, France. Since 2014, he has been a Post-Doctoral Fellow with the Biomedical Imaging Group, École Polytechnique Fédérale de Lausanne, Switzerland, and the Center for Biomedical Imaging, Lausanne University. His main research interests include inverse problems for image reconstruction, motion estimation and image analysis for fluorescence microscopy.

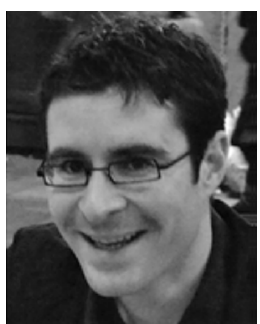

Paul Guichard received the Ph.D. degree in 2010 in cell biology and biophysics from the University of Pierre and Marie Curie, Paris, France. He did his postdoctoral training in the Lab of Pierre Gonczy at the Swiss Federal Institute of Technology, Lausanne, Switzerland. He's now currently an Assistant Professor at the University of Geneva. His main research area is structural biology of cellular components.

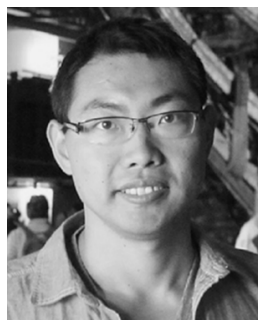

Ning Chu received the M.Sc. degree in automatic, signal, and image processing from the University of Paris Sud, France, in 2010, and the Ph.D. degree in signal and image processing in 2013 from the University of Paris Sud, Supélec, France. From 2014 to 2015 , he was a Post-Doctoral Fellow with the Biomedical Imaging Group, École Polytechnique Fédérale de Lausanne, Switzerland. His research interests mainly focus on acoustic source imaging and inverse problem applied in biomedical imaging. 




Michael Unser (M'89-SM'94-F'99) received the M.S. (summa cum laude) and Ph.D. degrees in electrical engineering from the cole Polytechnique Fédérale de Lausanne (EPFL), Switzerland, in 1981 and 1984 , respectively. From 1985 to 1997 , he was a Scientist with the National Institutes of Health, Bethesda, USA. He has a strong interest in sampling theories, multiresolution algorithms, wavelets, and the use of splines for image processing. He has authored 200 journal papers on those topics, and is one of ISIs highly cited authors in engineering. He is currently a Full Professor and the Director of the Biomedical Imaging Group with EPFL. His main research area is biomedical image processing. He was the
Associate Editor-in-Chief of the IEEE TRANSACTIONS ON MEDICAL IMAGING (2003-2005), and served as an Associate Editor of the IEEE TRANSACTIONS ON MEDICAL IMAGING (1999-2002 and 2006-2007), the IEEE TRANSACTIONS ON IMAge Processing (1992-1995), and the IEEE SignAL Processing LeTters (1994-1998). He is currently a member of the Editorial Boards of the IEEE Journal of SELECTED TOPICS IN SIgNAL PROCESSING and SIAM Journal on Imaging Sciences. He co-organized the first IEEE International Symposium on Biomedical Imaging (2002), and was the Founding Chair of the Technical Committee of the IEEE-SP Society on Bio Imaging and Signal Processing. He was a recipient of the 1995 and 2003 best paper awards, the 2000 Magazine Award, and two IEEE Technical Achievement Awards (2008 SPS and 2010 EMBS). He is a fellow of the European Association for Signal Processing and a member of the Swiss Academy of Engineering Science. 